\title{
Heart failure with mid-range ejection fraction: A review of clinical status and meta-analysis of clinical management methods
}

\author{
Aref Albakri* \\ St-Marien hospital Bonn Venusberg, Department of internal medicine, Bonn, Germany
}

\begin{abstract}
Traditionally, heart failure (HF) has been defined based on pump dysfunction and classified based on left ventricular (LV) ejection fraction (LVEF) - HF with reduced $\mathrm{EF}$ (HFrEF: $\mathrm{EF}<40 \%$ ) and HF with preserved EF (HFpEF: EF > 50\%). In 2016, the ESC Guidelines proposed a new HF phenotype termed HF with midrange ejection fraction (HFmEF) referring to $\mathrm{HF}$ patients with mildly reduced (borderline) ejection fraction of $40 \%$ to $49 \%$. However, research on $\mathrm{HFmEF}$ is scarce and highly fragmented. It consists largely of post hoc analysis of clinical registries and sub-analysis of population-based studies. The few research on $\mathrm{HFmEF}$ focus on comparison of clinical characteristics, comorbidities, prognosis and outcomes between HFmEF, HFrEF and HFpEF. To provide a holistic understanding of HFmEF, the present review synthesizes key findings from published literature on epidemiology, etiology, pathophysiology, diagnosis and clinical management of HFmEF.
\end{abstract}

\section{Introduction}

The initial recognition of heart failure (HF) with mid-range ejection fraction (HFmEF) appeared in the 2012 ESC guidelines for the diagnosis and treatment of acute and chronic heart failure. The guidelines recognized a grey area in the classification of patients based on left ventricular ejection fraction (LVEF) of 40-49\% [1]. Similarly, the 2013 American College of Cardiology/American Heart Association (ACC/AHA) guidelines for the management of heart failure reported patients with $\mathrm{LVEF}$ of $40-50 \%$ represent an intermediate group between $\mathrm{HF}$ with reduced (HFrEF) and preserved ejection fractions (HFpEF) [2]. The 2016 ESC heart failure guidelines termed the phenotype HFmEF [3]. The reason for the creation of the HFmEF phenotype was not based on a new group of patients rather to provide a formal nomenclature and stimulate research on HFmEF [3]. The prevailing question is whether the HFmEF phenotype represent a distinct pathophysiological entity or is merely a transitional phenotype between HFrEF and HFpEF. The search for an answer to this question is ongoing and hoped to determine the efficacy of the clinical management strategies for HFmEF patients. This review article provides a systematic discussion of observational studies and post hoc analysis of large clinical registries.

\section{History, definition and epidemiology \\ History}

Pump dysfunction (the inability of the heart to eject sufficient blood to meet metabolic demands of body organs and tissues) remains the cornerstone method of defining, classifying and treating heart failure (HF) patients. The predominant method of measuring pump dysfunction is the left ventricular ejection fraction (LVEF) defined as the proportion of diastolic volume ejected during ventricular contraction with $55 \%$ to $75 \%$ considered the normal range [4]. Based on LVEF values, HF has been classified into three phenotypes: HF with reduced ejection fraction (HFrEF: LVEF < 40\%), HF with mid-range ejection fraction (HFmEF: LVEF 40-49\%) and HF with preserved ejection fraction (HFpEF: $L V E F \geq 50 \%$ ) [5]. The utility of LVEF in classifying HF patients has received criticism but its use will continue to dominate HF research and practice because it forms the basis for the current risk-stratification, pathophysiology, diagnosis, and treatment guidelines and protocols for HF patients $[5,6]$. Whereas the intention of introducing the HFmEF phenotype was to stimulate research, the low prevalence of $\mathrm{HFmEF}$ poses a major challenge for conducting largescale clinical trials. Already, the MIRACLE EF and MADIT-ASIA trials were terminated due to enrolment futility. The MIRACLE EF trial had enrolled only 44 patients after 13 months [7].

\section{Definition}

HFmEF is the latest described phenotype of HF. The 2016 ESC heart failure guidelines define HF as follows. It is "a clinical syndrome characterized by typical symptoms of breathlessness, ankle swelling and fatigue that may be accompanied by signs such as elevated jugular venous pressure, pulmonary crackles and peripheral edema caused by a structural and/or functional cardiac abnormality resulting in a reduced cardiac output and/or elevated intra-cardiac pressures at rest or during stress" [3]. The 2013 ACC/AHA heart failure guidelines defines HF as, "a complex clinical syndrome that results from any structural or functional impairment of ventricular filling or ejection of blood". The cardinal clinical manifestations include dyspnea and fatigue limiting exercise tolerance and fluid retention leading to pulmonary and/or

${ }^{\star}$ Correspondence to: Aref Albakri, St-Marien hospital Bonn Venusberg, Department of internal medicine, Bonn, Germany, E-mail: arefalbakri@yahoo.com

Key words: heart failure with mid-range ejection fraction, borderline ejection fraction, mildly reduced ejection fraction

Received: November 02, 2018; Accepted: November 15, 2018; Published: November 19, 2018 
splanchnic congestion and/or peripheral edema [2]. Drawn from the definition of HF, the 2016 ESC guidelines define HFmEF as clinical syndrome presenting with typical HF signs and/or symptoms, LVEF of 40-49\%, elevated levels of serum natriuretic peptides and presence of structural heart disease or diastolic dysfunction [3]. In sum, HFmEF encompasses all patients with a clear diagnosis of HF by biological and/ or imaging criteria distinguished by LVEF of $40-49 \%$.

\section{Epidemiology}

The HFmEF phenotype lacks focused population-based epidemiological studies. However, sub-analysis of clinical registries and community-based studies provide an estimation of its prevalence and incidence relative to the HFrEF and the HFpEF phenotypes. In the U.S., the percentage of the HF population within the HFmEF category ranges between $13-24 \%$ translating into about 1.6 million individuals [8]. A handful of studies report HFmEF prevalence of between $10 \%$ and $20 \%$ of all HF patients [9-12]. The Get With The Guidelines-HF (GWTG$\mathrm{HF}$ ) registry provides the largest cohort to date characterizing HFmEF $[13,14]$. In the most recent analysis of GWTG-HF registry involving 99,825 patients between 2005 and 2013, HFmEF accounted for 13\% of all hospitalized HF patients [14]. In the European Society of Cardiology Heart Failure Long-Term (ESC-HF-LT) Registry [15] and the Swedish HF Registry [16,17], HFmEF accounted for $24 \%$ and $21 \%$ respectively of all the hospitalized HF patients (Table 1). In terms of age and sex, the GWTG-HF registry reveals HFmEF patients are older (median age 77 years) and with an almost equal gender representation, female (49\%). However, the ESC-HF-LT and Swedish registries indicate patients with HFmEF are older (64 and 74 years) and more likely to be male $(68.5 \%$ and $61 \%)$.

\section{Comorbidity burden}

The precise knowledge and understanding of the burden of comorbidities on HFmEF patients remain incompletely understood with a majority of studies focusing only on a comparative analysis with HFrEF and HFpEF. Further, with the conspicuous lack of supporting evidence from clinical trials, information about comorbidities are only available from large registries, which are based in different geographic regions and thus provide highly variable and less consistent findings about incidence of cardiac and extra cardiac comorbidities in HFmEF. In addition, although registries are important to provide large datasets for comparison, the data may not reflect the actual comorbidity burden encountered in a clinical setting. In a comparison of comorbidities burden between HFmEF, HFrEF and HFpEF phenotypes in three (3) large registries: GWTG-HF [14], ESC-HF-LT [15], and Swedish HF [17] (Table 2), HFmEF has a high hypertension comorbidity burden $(82.2 \%$, $60.1 \%$ and 64.0 ), which is intermediate between HFrEF $(77.8 \%, 55.6 \%$ and $56.0 \%)$ and HFpEF (84.0\%, 67.0\% and 72.0\%). HFmEF also has a high CAD comorbidity burden (59.9\%, 41.8\% and 53.0\%) comparable to $\mathrm{HFrEF}(58.4 \%, 48.6 \%$ and $54.0 \%)$ but much higher than HFpEF (48.6\%, 23.7\% 42.0\%). The burden of diabetes and atrial fibrillation is higher in HFmEF than in the HFrEF phenotype but comparable to the HFpEF phenotype but previous myocardial infarction and revascularization procedures are more common in $\mathrm{HFmEF}$ and HFrEF phenotypes than in the HFpEF phenotype. However, the Trial of Intensified versus standard Medical therapy in Elderly patients with Congestive Heart Failure (TIME-CHF) registry finds a comparable higher burden of comorbidities in all the three HF phenotypes [18]. The varying comorbidity burden between large registries underscore the need of randomized clinical trials to determine the actual incidence of comorbidity in HFmEF phenotype.

\section{Prognosis}

\section{Predictors, mortality and hospitalization}

Community/multi-center studies: The evidence of the prognostication of HFmEF is still in its nascent stage with divergent outcomes. Bhambhani and associates [19] pooled data from four community-based longitudinal cohorts involving 28,200 patients, with HFmEF accounting for $10 \%$ of all new HF cases within a twelveyear period. They found significant prognostic predictors of HFmEF included older age, male gender, higher systolic blood pressure, presence of diabetes mellitus, and a history of myocardial infarction (MI). Significant biomarkers predicting HFmEF were natriuretic peptides, cystatin- $\mathrm{C}$ and troponin. The same biomarkers had a higher correlation with HFrEF incidence. The onset of HFmEF had a higher rate of allcause mortality (50 events per 1,000 person-years) compared to the

Table 1. Prevalence of HFmEF, HFrEF and HFpEF in large registries

\begin{tabular}{|c|c|c|c|c|c|c|}
\hline Registry & \multicolumn{2}{|c|}{ HFmEF } & \multicolumn{2}{c|}{ HFrEF } & Total \\
\hline & N & $\%$ & N & $\%$ & N \\
\hline GWTG-HF [13,14] & 12,918 & 13 & 48,950 & 49 & 38,056 \\
\hline ESC-HF-LT [15] & 2,212 & 24 & 5,460 & 60 & 1,462 \\
\hline Swedish HF [16,17] & 8,374 & 21 & 22,981 & 57 & 99,825 \\
\hline
\end{tabular}

ESC-HF-LT: European Society of Cardiology Heart Failure Long-Term; GWTG-HF: Get With The Guidelines-HF; N: Total

Table 2. Comorbidities Burden in HFmEF, HFrEF and HFpEF Based on Clinical Registries

\begin{tabular}{|c|c|c|c|c|c|c|c|c|c|}
\hline \multirow[b]{2}{*}{ Comorbidities } & \multicolumn{3}{|c|}{ GWTG-HF [14] (\%) } & \multicolumn{3}{|c|}{ ESC-HF-LT [15] (\%) } & \multicolumn{3}{|c|}{ Swedish HF [17] (\%) } \\
\hline & HFrEF & HFmEF & HFpEF & HFrEF & HFmEF & HFpEF & HFrEF & HFmEF & HFpEF \\
\hline Anemia & 19.0 & 27.0 & 29.2 & - & - & - & 31.0 & 35.0 & 41.0 \\
\hline $\mathrm{AF}$ & 34.2 & 41.8 & 42.5 & 18.3 & 22.3 & 32.2 & 51.0 & 58.0 & 63.0 \\
\hline CAD & 58.4 & 59.9 & 48.6 & 48.6 & 41.8 & 23.7 & 54.0 & 53.0 & 42.0 \\
\hline COPD/Asthma & 31.9 & 36.4 & 39.8 & 15.2 & 11.6 & 14.0 & - & - & - \\
\hline Diabetes & 44.5 & 50.2 & 49.0 & 32.3 & 30.5 & 29.3 & 27.0 & 27.0 & 28.0 \\
\hline Stroke/TIA & 15.9 & 17.1 & 17.2 & 9.4 & 8.3 & 9.8 & - & - & - \\
\hline Hypertension & 77.8 & 82.2 & 84.0 & 55.6 & 60.1 & 67.0 & 56.0 & 64.0 & 72.0 \\
\hline Previous MI & 30.6 & 25.5 & 16.7 & - & - & - & - & - & - \\
\hline CKD & 23.1 & 25.8 & 24.5 & 19.5 & 16.5 & 19.9 & 45.0 & 48.0 & 56.0 \\
\hline
\end{tabular}

AF: Atrial Fibrillation; CAD: Coronary Artery Disease; COPD: Chronic Obstructive Pulmonary Disease; MI: Myocardial Infarction 
onset of HFpEF (39 events per 1,000 person-years) but comparable to that of HFrEF (46 events per 1,000 person-years; $\mathrm{p}=0.78$ ) [19].

In Catalonia (Spain), a large multi-center prospective observational study recruited 3,580 HF patients from four university hospitals dedicated to HF. The sample included $14 \%$ patients with HFmEF. In a median follow-up of 3.66 years, all-cause mortality was lower in the HFmEF phenotype (43.8\%) compared to HFrEF (45.8\%) and HFpEF (52.6\%). Hospitalization due to HF, and cardiovascular death did not differ between HFmEF and HFrEF phenotypes but lower than HFpEF [20].

Clinical registries: Sub-analysis of clinical registries also provide important insights into prognostication of HFmEF more so in comparison to HFrEF and HFpEF but with divergent findings.

The ESC-HF-LT [15] one-year follow-up data in 9,134 ambulatory HF patients including $24.2 \%$ with HFmEF phenotype reveals no significant differences in all-cause mortality with HFrEF and HFpEF phenotypes but non-cardiovascular mortality (27.8\%) was higher than HFrEF (20.1\%) but slightly lower than HFpEF (30.7\%) but the difference was not significant $(\mathrm{p}=0.06)$. HFmEF had the lowest rate of hospitalization (8.7\%) compared to HFrEF (14.6\%) and HFpEF (9.7\%) [15].

The GWTG-HF registry recruiting 99,825 hospitalized HF patients, pneumonia was associated with longer hospital stay while dietary and medication non-compliance with reduced length of stay. In-hospital mortality rates was lower in the HFmEF phenotype (2.6\%) compared to HFpEF (3.2\%) and HFrEF (3.0\%) [14].

The Swedish HF Registry included 42,061 hospitalized and ambulatory HF patients with HFmEF constituting $21 \%$. All-cause mortality at one, twelve and thirty-six months was higher in HFmEF and HFpEF than HFrEF at all the three points but the difference was not statistically significant. However, in the presence of CAD, HFmEF had a higher three-year mortality rate [16].

The Acute Heart Failure Global Registry of Standard Treatment (ALARM-HF) involving 4,953 hospitalized HF patients, HFmEF accounted for $25 \%$ of those with documented LVEF $(n=3,257)$. Compared to HFrEF and HFpEF, the most frequent and significant causes of hospitalization for HFmEF were acute coronary syndrome (38.6\%) or infection (17\%) [21].

The National Spanish RICA Registry involving 2,753 hospitalized HF patients $(H F m E F=10.2 \%)$, HFmEF had significantly lower oneyear mortality rate (20\%) compared to HFrEF (28\%) and HFPEF (22\%). There was no significant difference in both 30-day and one-year rehospitalization rates in all the three HF phenotypes [22].

Two Spanish registries (REDINSCOR I and MUSIC) with a combined 3,446 ambulatory HF patients (13.3\% diagnosed with HFmEF) followed for a period of 41 months, all-cause mortality in HFmEF (27\%) and HFpEF (28\%) were significantly lower compared to HFrEF (33\%). The risk of cardiovascular death did not differ between HFmEF and HFrEF but HFmEF had a significantly higher risk of cardiovascular death and sudden cardiac death than HFpEF [23].
The Spanish Network for the Study of Heart Failure (REDINSCOR II) Registry, where HFmEF accounted for $16 \%$ of patients hospitalized with de novo or decompensated HF $(n=1,420)$, there was no significant difference in all-cause mortality and re-admission rates in all the three HF phenotypes [24].

In summary, data from large clinical registries reveal divergent and inconsistent findings of prognostication (all-cause mortality, cardiovascular mortality and re-hospitalization) of HFmEF in comparison to HFrEF and HFpEF. However, several factors could contribute to the divergence. These include (a) the HF patient population included such as acute HF, stable HF or a combination of the two and ambulatory or hospitalized HF patients; (b) patient characteristics such as age, gender, and race and presence/absence of comorbidities; and (c) various periods of follow-up ranging from one (1) month to three (3) years. These factors have been reported to affect the epidemiology of HFmEF [25-28]. Despite the variation in prognosis, in overall, the medium-term prognosis of HFmEF appears intermediate to HFrEF and HFpEF while long-term prognosis HFmEF might be similar to that of HFpEF. However, the differences warrant clinical trials to determine the long-term prognosis of HFmEF phenotype rather than just a comparison with HFrEF and HFpEF.

\section{Changes in ejection fraction and prognosis}

Temporal changes in ejection fraction: The LVEF in HF usually shifts from one phenotype to another with the HFmEF phenotype having the highest rate of transitioning LVEF. A multi-center study in Catalonia Spain reports after one-year follow-up, the HFmEF phenotype registered the highest variability in LVEF measures: $24 \%$ had depressed LVEF (moved to HFrEF), 43\% maintained LVEF (remained HFmEF) while the remaining 33\% improved LVEF (moved to HFpEF) [20]. Similar temporal changes in LVEF in HFmEF patients has been observed in clinical registries (Table 3 ).

In the Swedish HF registry, about $25 \%$ of HFmEF patients transitioned into HFpEF and more than 33\% transitioned into HFrEF with ischemic heart disease (IHD) cited as a key cause of worsening LVEF [17]. In the Chronic Heart Failure Analysis and Registry in the Tohoku District-2 (CHART-2) study, at one-year, $44 \%$ of HFmEF patients transitioned to HFpEF and $16 \%$ to HFrEF. The Washington University Heart Failure Registry had the highest HFmEF transitioning rates of $90 \%$ constituting $73 \%$ to $\mathrm{HFpEF}$ and $17 \%$ to HFrEF [30]. The high rate of HFmEF transitioning to either HFrEF or HFpEF raises the question whether it is a distinct phenotype of HF or a transition between HFrEF and HFpEF phenotypes.

Effect of transitioning LVEF on prognosis: Transitioning LVEF from HFmEF to either HFrEF or HFpEF has been associated with worsening or favorable prognosis depending on the direction of transition. Increases in LVEF values suggest recovery (improving outcomes) while decreasing LVEF values suggest deterioration of symptoms ow worsening disease progression (Figure 1).

Studies on HFmEF LVEF changes indicated improvement with transition to HFpEF and deterioration of clinical outcomes with

Table 3. Temporal Changes in LEVF in HFmEF Patients at 1-Year (Registry Data)

\begin{tabular}{|l|c|c|c|}
\hline Clinical Registry & Worsened (Transitioned to HFrEF) & Remained Stable (Remained at HFmEF) & Improved (Transitioned to HFpEF) \\
\hline Swedish HF [17] & $33 \%$ & $42 \%$ \\
\hline CHART-2 [29] & $16 \%$ & $40 \%$ \\
\hline $\begin{array}{l}\text { Washington University Heart Failure } \\
\text { Registry [30] }\end{array}$ & $17 \%$ & $10 \%$ & $44 \%$ \\
\hline
\end{tabular}

CHART-2: Chronic Heart Failure Analysis and Registry in the Tohoku District-2 


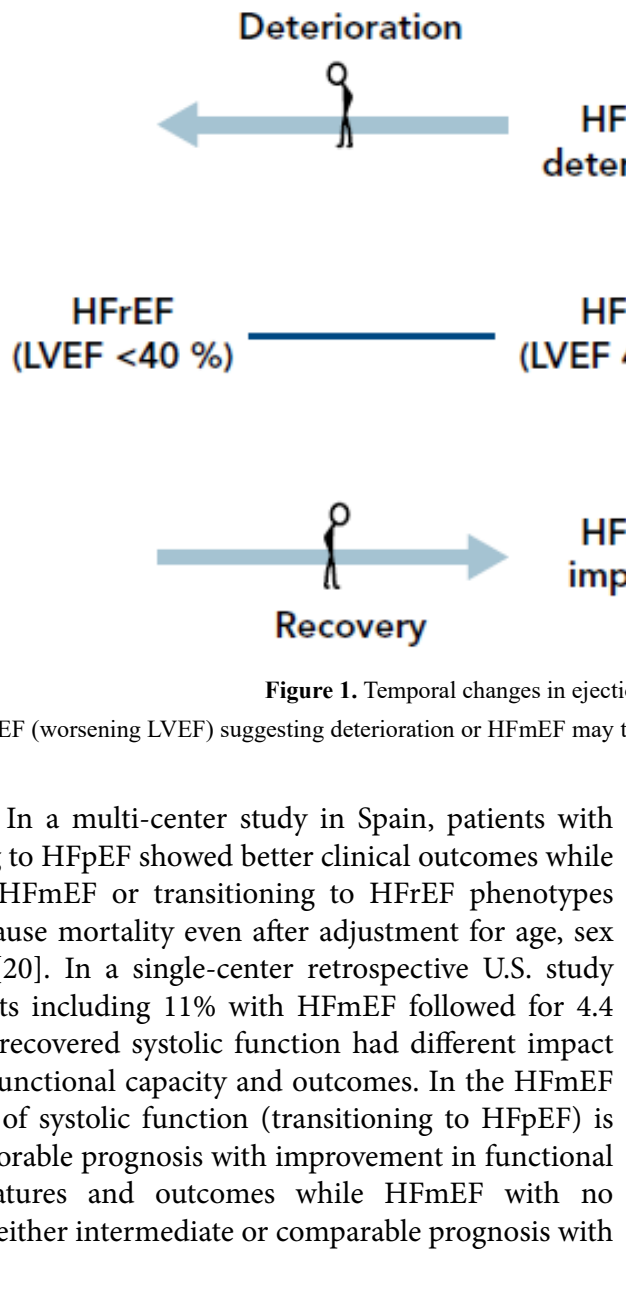
HFpEF [31]

Data from registries supported the worsening clinical outcomes for HFmEF patients transitioning to HFrEF and vice-versa to HFpEF. The Swedish HF registry [17]. In CHART-2 registry, after one-year follow-up HFmEF patients had comparable mortality rates to HFpEF patients, which was better than that of HFrEF. HFmEF at registration who transitioned to HFrEF had increased mortality at one-year follow-up [29]. The Washington University Heart Failure Registry showed significant improvement in clinical outcomes (mortality, heart transplantation, HF hospitalization, HF-related hospitalization) when HFmEF transitioned to HFpEF relative to matched patients with HFrEF and HFmEF transitioning to HFrEF [30].

In a study of the natural history of left ventricular ejection fraction in patients with heart failure, Clarke, et al. [32] examined LVEF transitions between HFpEF and HFrEF. The study recruited involved 2,413 patients in Colorado between 2001 and 2008 with a mean followup of 4.4 years. They were 8,813 transitions to suggest LVEF is a dynamic factor influenced by sex of the patients, presence of comorbidities, and drug therapy. Women are more likely to transit from HFrEF to HFpEF (Hazard Ratio [HR], 1.85; 95\%; CI: 1.38-2.47), and patients adherent to beta-blocker therapy are more likely to transit from HFrEF to HFpEF (HR, 1.53; 95\%; CI: 1.10-2.13). On the other hand, HFpEF patients with myocardial infarction were more likely to transition to HFrEF (HR, 1.75; 95\%; CI: 1.26-2.42) [32]. Although the study did not use the current ESC guidelines on the classification of HFrEF, HFmEF and
HFpEF, it is expected that HFmEF will transition to HFrEF or HFpEF during sufficiently long follow-up period.

\section{Etiology and pathophysiology}

Etiology: The underlying etiology of HF is similar across the three phenotypes (HFrEF, HFmEF and HFpEF). Patients with HFmEF are more likely to show higher valvular heart disease and hypertensive etiology compared to HFrEF patients. These are typical etiologies in the HFpEF phenotype suggesting HFmEF is closer to HFpEF in terms of hypertension etiology. On the other hand, the HFmEF phenotype is similar to HFrEF with regard to ischemic etiology [30]. Patients with HFmEF and HFrEF have higher prevalence of diabetes, ischemic heart disease and idiopathic dilated cardiomyopathy (Figure 2). Lam and Solomon [10] postulate that based on current evidence on etiology, HFmEF may be a subset of HFpEF that acquires CAD and transitioning to HFrEF [10]. However, current evidence on etiology may suggest HFmEF is an intermediate phenotype to HFrEF and HFpEF since it shares ischemic etiology with HFrEF and hypertensive etiology with HFpEF.

Pathophysiology: Focused research on the pathophysiology of HFmEF is lacking. Nascent research has focused on analysis of changes in biomarker levels. In a study of 37 biomarkers, Tromp, et al. [33] investigated biomarkers levels in different pathophysiological domains: myocardial stretch, inflammation, angiogenesis, oxidative stress and hematopoiesis. The study reports that BNP levels in HFmEF (397 pg/ $\mathrm{ml})$ lie between those of HFrEF $(521 \mathrm{pg} / \mathrm{ml})$ and HFpEF $(300 \mathrm{pg} / \mathrm{ml})$ phenotypes suggesting an upward trend with decreasing LVEF. The study related biomarker interaction in HFrEF with cardiac stretch (remodeling), and HFpEF with inflammation. In HFmEF, biomarker interactions relate to both inflammation and cardiac stretch. In HFmEF and HFpEF, markers for remodeling at admission and alterations in levels for inflammatory markers in the first 24 hours predict all-cause mortality and re-hospitalization at two months [33]. A sub-analysis of the Singapore Heart Failure Outcomes and Phenotypes (SHOP) cohort of 1,096 HF in/outpatients reveals troponin levels in HFmEF lie between those of HFrEF and HFpEF [34]. In a prospective sub-analysis of the Swedish HF patients, Savarese et al. associates decreases in NT- 


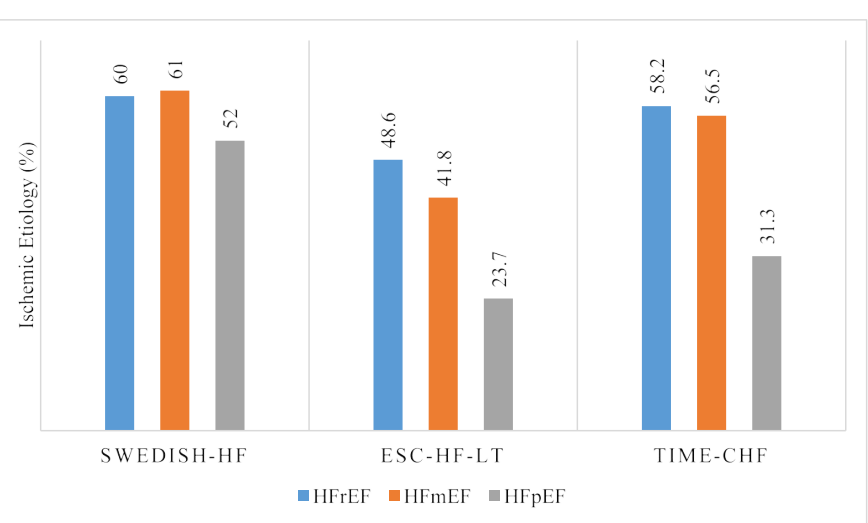

Figure 2: Ischemic etiology in HFrEF, HFmEF and HFpEF in clinical registries

Ischemic etiology in HFmEF and HFrEF phenotypes are comparable, having a much higher percentage compared to the HFpEF phenotype indicating HFmEF etiology is closer to that of HFrEF than to HFpEF

proBNP over time with better survival in all the three HF phenotypes [35].

\section{Clinical presentation and diagnosis}

Clinical presentation: The 2016 ESC heart failure guidelines highlight the cardinal HF symptoms are dyspnea, fatigue, and peripheral edema and cardinal HF signs are elevated jugular venous pressure, pulmonary crackles and peripheral edema [3]. However, these clinical signs and symptoms are general and non-specific for the three HF phenotypes: HFrEF, HFmEF and HFpEF. Irrespective of the HF phenotype, some patients may present with exercise intolerance but with little evidence of fluid retention (peripheral edema) while others may complain of edema, dyspnea or fatigue [2]. The prevalence of HF signs and symptoms is high in all the three phenotypes. The most recent analysis of the GWTG-HF registry provides valuable insights into the prevalence of cardinal HF signs and symptoms encountered during admission of HFrEF, HFmEF and HFpEF (Table 4).

Despite the GWTG-HF registry-based prevalence of HF signs and symptoms indicates at least one sign is common to over $93 \%$ in all the three phenotypes, the prevalence of signs and symptoms in HFmEF resembles that of HFpEF in acute pulmonary edema, sustained ventricular arrhythmias, pulmonary congestion and worsening fatigue. Similarly, in the ALARM-HF registry, the most common clinical presentation of $\mathrm{HFmEF}$ at admission are acute pulmonary edema, acute de novo HF or atrial fibrillation [21]. Most recent analysis of the GWTG-HF registry also reveals that most common precipitating factors for HF hospitalization in regardless of the HF phenotype are pneumonia/respiratory process (28\%), arrhythmia (22\%), medication noncompliance (16\%), worsening renal failure (15\%), and uncontrolled hypertension (15\%). Pneumonia had an independent association with prolonged hospital stay and higher in-hospital mortality regardless of the phenotype. Precipitating factors for hospitalization of HF patients differed across the three phenotypes (Figure 3).

Relative to the HFrEF and the HFpEF phenotypes, the most likely reasons for hospitalization of HFmEF patients are arrhythmias, uncontrolled hypertension and renal failure. Factors intermediate to HFrEF and HFpEF that most likely caused hospitalization of HFmEF patients are respiratory, medication non-compliance, diet noncompliance, and ischemia or acute coronary syndrome.

Diagnosis: There is no single gold standard method for diagnosis of HF, which usually require a series of tests. The 2016
ESC HF guidelines recommend a general diagnostic work-up for all HF phenotypes, which should include patient history, physical examination, laboratory tests and imaging tests (Figure 4).

From the general diagnostic work-up, LVEF values provide a distinction between the three HF phenotypes. The ESC guidelines recommend that the diagnosis of HFmEF should fulfil the following four elements:

a) Clinical symptoms of HF with or without signs;

b) Mildly depressed LVEF ( $40 \%$ to $49 \%$ );

c) Elevated levels of natriuretic peptides (BNP $\geq 35 \mathrm{pg} / \mathrm{mL}$ or NTproBNP $\geq 125 \mathrm{pg} / \mathrm{mL}$ ); and

d) At least one evidence of relevant structural heart disease:

- The presence of LV hypertrophy (LV mass index > $115 \mathrm{~g} / \mathrm{m}^{2}$ (males) and $95 \mathrm{~g} / \mathrm{m}^{2}$ (females) or

- Diastolic dysfunction (E/e' $\geq 13$ and mean e' septal and lateral wall $<9 \mathrm{~cm} / \mathrm{s}$ ).

Although there is a lack of clinical trials to confirm the accuracy of the ESC diagnostic work-up of HFmEF, a 2009 Chinese study supports the ESC-described echocardiography profile of HFmEF patients. The study compared ventricular structure and function in HF patients with $\mathrm{EF} \geq 55 \%$ versus those with LVEF $40 \%$ to $55 \%$ and those with LEVF $\leq 40 \%$ using non-invasive pressure volume analysis and revealed important pathophysiological differences between the three patient

Table 4. Prevalence of Clinical Manifestation in HFrEF, HFmEF and HFpEF

\begin{tabular}{|l|c|c|c|c|}
\hline \multicolumn{1}{|c|}{ Clinical Sign/Symptom } & $\begin{array}{c}\text { HFrEF } \\
\mathbf{( \% )}\end{array}$ & $\begin{array}{c}\text { HFmEF } \\
\mathbf{( \% )}\end{array}$ & $\begin{array}{c}\text { HFpEF } \\
\mathbf{( \% )}\end{array}$ & P value \\
\hline Acute pulmonary edema & 2.18 & 2.39 & 2.34 & 0.17 \\
\hline Syncope/dizziness & 3.09 & 2.08 & 2.15 & $<0.0001$ \\
\hline Dyspnea & 68.69 & 73.98 & 73.49 & $<0.0001$ \\
\hline ICD shock/ sustained ventricular arrhythmias & 0.53 & 0.18 & 0.10 & $<0.0001$ \\
\hline Pulmonary congestion & 3.51 & 3.25 & 3.38 & 0.29 \\
\hline Volume overload/weight gain & 11.75 & 9.99 & 11.05 & $<0.0001$ \\
\hline Worsening fatigue & 3.27 & 2.63 & 2.49 & $<0.0001$ \\
\hline $\begin{array}{l}\text { Count of Symptoms/Signs (at least 1 sign } \\
\text { present) }\end{array}$ & 93.02 & 94.48 & 95.01 & $<0.0001$ \\
\hline
\end{tabular}

ICD: Implantable Cardioverter-Defibrillator

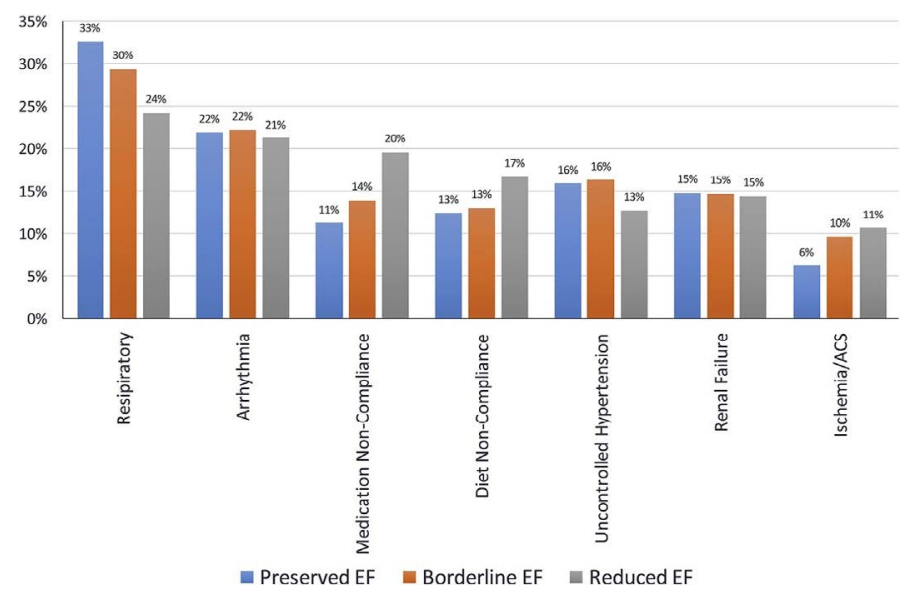

Figure 3. Precipitating factors for HF admission based on HF phenotypes

Patients with HFmEF were more likely to have worsening or life-threatening arrhythmias, uncontrolled hypertension and renal failure compared with those with HFrEF. ACS: Acute Coronary Syndrome; EF: Ejection Faction. Adapted from Kapoor et al., (GWTG-HF registry), 2017, p 


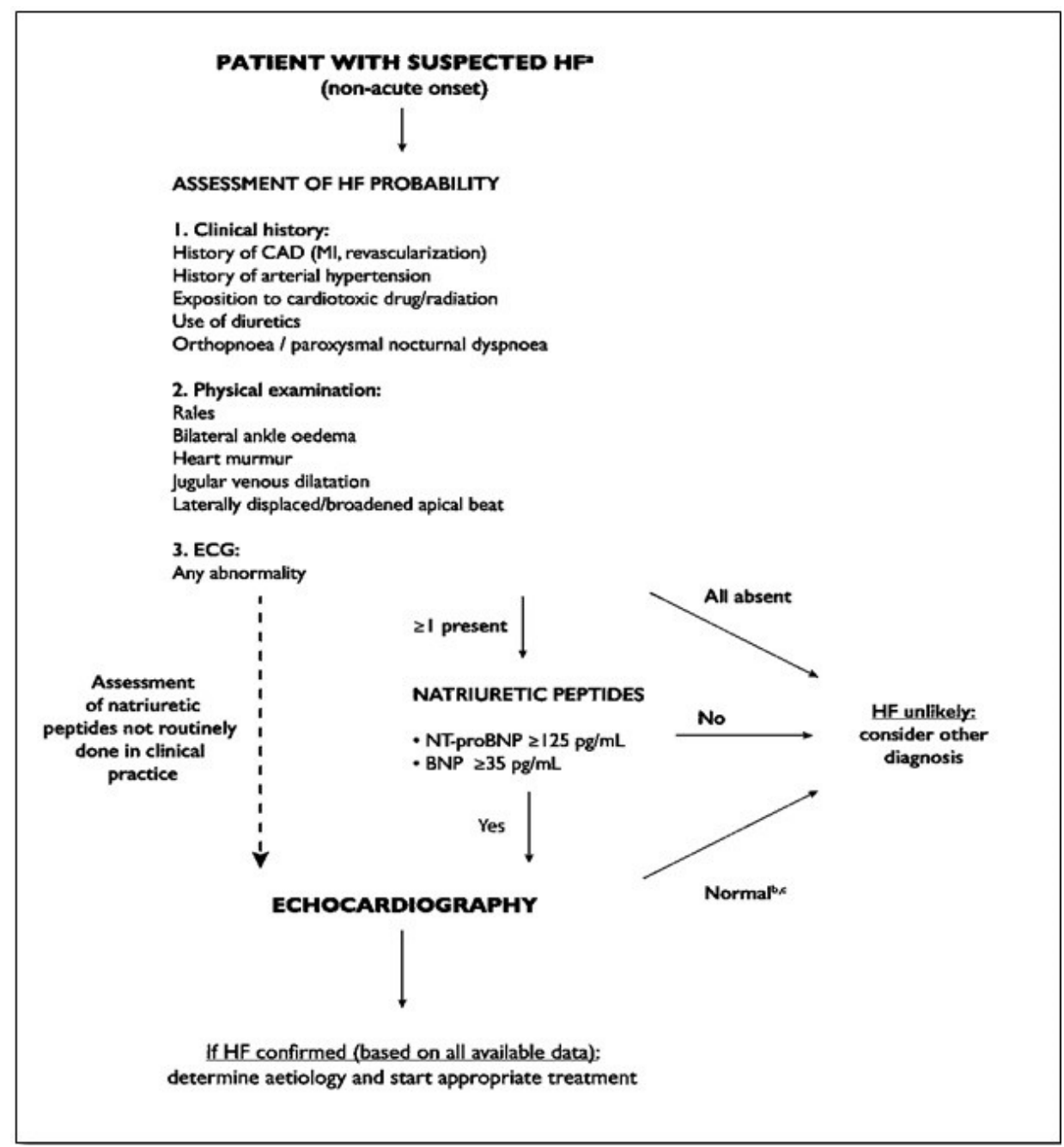

Figure 4. Diagnosis work up for HF in a non-acute setting

Diagnosis of HFmEF begins with assessment of clinical history and physical examination to identify risk factors, and signs and symptoms respectively as well as ECG abnormalities. If all are absent, HFmEF is unlikely, but is any sign or symptom is present, assessment of the level sof natriuretic peptides or echocardiography should be considered to confirm the diagnosis of HFmEF

groups. HF patients with LVEF 40-55\% exhibited increased LV diastolic stiffness same to those with LVEF $\geq 55 \%$ but also revealed significant alterations in ventricular size and function similar to patients with LEVF $\leq 40 \%$. The LVEF $40-50 \%$ group had significant enlargement of the LV by eccentric remodeling and a marked decrease in chamber contractility. Diastolic dysfunction and LA enlargement were common in all the three groups of HF patients with most severe cases in the LVEF $\leq 40 \%$ patient group. The findings are consistent with the ESC recommendations on the diagnosis of HFmEF indicating diastolic dysfunction, hypertrophied LV and systolic dysfunction [36].

\section{Clinical management}

Management guidelines: Previous ESC heart failure guidelines established only two phenotypes of HF, HFrEF defined by LVEF $\leq$ $40 \%$ and HFpEF defined by LVEF > 50\% [37]. However, many clinical trials investigating different clinical management strategies on HFrEF included patients with LVEF $<35 \%$ to $40 \%$ and those on HFpEF included patients with LVEF > 45\% [38-40]. The result was HFmEF patients were not well represented in clinical trials of HF [26]. The Current ESC HF guidelines however recommend therapy for HFmEF patients based on evidence for HFpEF than for HFrEF. In addition, the guidelines recommend diuretic therapy for HFmEF patients with congested HF for the relief of symptoms and signs [3]. Patients with HFmEF also have a high prescription rate of the traditional HF medication such as angiotensin-converting enzyme (ACE)-inhibitors, angiotensin receptor blockers (ARBs), mineralocorticoid receptor antagonists (MRAs) and beta-blockers [25].

Meta-analysis of clinical management methods: Clinical management of HFmEF lacks both specific guidelines and clinical trials that assess treatment efficacy and/or prognostication. However, research reports suggest a high number of HFmEF patients receive the traditional HF pharmacological and non- pharmacological therapies but the efficacy of the traditional HF therapies lacks evidence-based support from clinical trials. However, clinical registries have pertinent data on HFmEF patients. They enroll all patients with a particular illness and methods of clinical management over time to provide a picture closer to the real world [41]. Thus, the present meta-analysis seeks to combine findings from sub-analysis of clinical registries to determine the frequently used medication and devices in the treatment of HFmEF patients. 
Search criteria and inclusion: A systematic search for relevant subanalysis of large clinical registries were searched from online databases PubMed, Medline, EMBASE as well as Google Scholar. The inclusion criteria were studies that (a) analyzed clinical registries that included HFmEF patients (LVEF $40 \%$ to $49 \%$ ); (b) listed HF medication administered or prescribed to HFmEF patients; and (c) provided at least one clinical outcome (all-cause mortality, cardiovascular mortality or rehospitalization). Citations of all selected studies from the initial online search were reviewed to identify additional relevant studies. There was no restriction on publication period, region or language. For studies analyzing data from the same clinical registry, the latest study was included. Analysis of data focused on getting percentages of HF medication used on HFmEF patients in the selected registries to determine the prevalence of medical therapy in this HF phenotype. The abstracted data included the name of the clinical registry, HFmEF population and outcomes - all-cause mortality, cardiovascular mortality and hospital readmission (Table 5).

Study characteristics and outcomes: Online search and citation review yielded thirty-six (36) potential studies conducting sub-analysis of clinical registries of HF patients. After subjecting the studies to the inclusion/exclusion criteria, only eight studies were included in the present meta-analysis [15,16,22,23,24,29,42,43]. The eight studies carried out sub-analysis of eight clinical registries. ESC-HFLT [15], Swedish HF [16], RICA [22], MUSIC/ REDINSCOR I [23], REDINSCOR II [24], CHART-2 [29], GWTG-HF [42], and TIMECHF [43]. The combined total population of HF patients in the eight registries was 16,455 . The weighted average of the percentage of HFmEF patients receiving HF medication varied widely (Table 6). The most used HF medication was loop diuretics (85.98\%), beta-blockers $(83.47 \%)$, ACE-inhibitors (81.11\%) and diuretics (68.11\%). The least use HF medications were Ivabradine $(8.10 \%)$, digoxin $(16.28 \%)$, vasodilators (16.84\%) and nitrates (18.06\%). Device therapies using ICD and CRT were not common in HFmEF patients representing 3.83\% and $4.39 \%$ respectively.

Analysis of the effect of HF medication on hard clinical end-points (hospitalization and mortality) was not possible as few studies directly reported the incidence of hospital re-admission, all-cause mortality and HF-related mortality. Most studies provided a comparison of hospitalization and mortality between HFrEF, HFmEF and HFpEF. Reporting periods also varied widely ranging between one month

Table 5. Summary of Included Sub-Analysis of Clinical Registries

\begin{tabular}{|c|c|c|c|c|c|c|c|c|}
\hline & ESC-HF-LT [15] & Swedish HF [16] & RICA [22] & $\begin{array}{c}\text { MUSIC/ } \\
\text { REDINSCOR I [23] }\end{array}$ & $\begin{array}{l}\text { REDINSCOR } \\
\text { II [24] }\end{array}$ & CHART-2 [29] & GWTG-HF [42] & TIME-CHF [43] \\
\hline Population (HFmEF) & 2,212 & 8832 & 280 & 448 & 227 & 596 & 3,285 & 575 \\
\hline ACE-I/ARBs & 91.7 & 84.0 & 79.0 & 87.2 & 72.4 & 80.0 & 64.7 & 90.7 \\
\hline Beta-blockers & 92.9 & 86.0 & 71.0 & 76.7 & 71.8 & 63.8 & 78.5 & 73.1 \\
\hline MRAs & 67.8 & 24.0 & 41.0 & 40.0 & 45.0 & 29.3 & 11.9 & 33.3 \\
\hline Ivabradine & 9.0 & NR & 1.0 & NR & NR & NR & NR & NR \\
\hline Diuretics & 78.0 & 74.0 & NR & NR & NR & 63.3 & 46.5 & NR \\
\hline Loop diuretics & NR & NR & 93.0 & 76.7 & NR & NR & NR & 89.8 \\
\hline Digoxin & 19.0 & 16.0 & 25.0 & 16.8 & NR & NR & 14.8 & 13.9 \\
\hline Anticoagulation & NR & 38.0 & 53.0 & NR & NR & NR & 31.9 & NR \\
\hline Statins & NR & 48.0 & NR & 59.1 & NR & 39.6 & 55.4 & NR \\
\hline Platelet inhibitors & NR & 53.0 & 41.0 & 30.2 & NR & 27.0 & 45.4 & NR \\
\hline Nitrates & NR & 17.0 & NR & NR & NR & NR & 18.4 & 32.4 \\
\hline CRT & 8.4 & 0.9 & NR & 4.6 & NR & 1.8 & 11.5 & NR \\
\hline ICD & 13.4 & 1.3 & NR & 7.0 & NR & 3.9 & 3.9 & 2.8 \\
\hline Vasodilators & NR & NR & 29.0 & NR & NR & NR & 15.8 & NR \\
\hline 12-Month Readmission & 8.7 & NR & NR & NR & 29.5 & NR & 48.5 & NR \\
\hline 12-Month Mortality & 7.6 & NR & 20.0 & 27.8 & 24.2 & NR & 85.7 & 7.2 \\
\hline Cardiovascular Death & 3.8 & NR & NR & NR & 15.8 & NR & NR & 4.5 \\
\hline
\end{tabular}

ACE: Angiotensin-Converting Enzyme; ARB: Angiotensin Receptor Blockers; CRT: Cardiac Resynchronization Therapy; ICD: Implantable Cardioverter-Defibrillator; MRA: Mineralocorticoid Receptor Antagonist

Table 6. Weighted Average of Percentage of HFmEF Patients Receiving HF Medication

\begin{tabular}{|c|c|}
\hline Frequently Administered/Prescribed HF Medication & Percentage (\%) of Patients Receiving HF Medication (Weighted Average) \\
\hline Loop diuretics & 85.98 \\
\hline Beta-blockers & 83.47 \\
\hline ACE-I/ARBs & 81.11 \\
\hline Diuretics & 68.11 \\
\hline Statins & 49.84 \\
\hline Platelet inhibitors & 48.98 \\
\hline Anticoagulation & 36.72 \\
\hline MRAs & 29.00 \\
\hline Nitrates & 18.06 \\
\hline Vasodilators & 16.84 \\
\hline Digoxin & 16.28 \\
\hline Ivabradine & \\
\hline CRT & \\
\hline ICD & \\
\hline
\end{tabular}


and five-years, making analysis of the effect of medication difficult. However, the few studies that reported hard end-points reported HFrelated hospital re-admission rates at one-year ranged between $8.7 \%$ [15] and $29.5 \%$ [24] and went up to $48.5 \%$ at five years [42]. All-cause mortality at one year ranged between $7.2 \%$ [43] and $27.8 \%$ [23], and rose to $85.7 \%$ at five years [42]. HF-related deaths at one year ranged between $3.8 \%$ [15] and $15.8 \%$ [24].

\section{Discussion}

The 2016 ESC guidelines recommend clinical management of HFmEF should use therapeutic strategies validated for HFpEF patients. The present meta-analysis sought to determine the prevalence of the utility of the traditional HF medication on HFmEF patients based on data from sub-analyses of eight clinical registries. In the absence of clinical trials, registries provide valuable information of selected medical conditions and current clinical management strategies and thus, help in identifying problems such as medication adherence, side effects, and treatment efficacy [41]. The present analysis finds use of HF medication such as diuretics, ACE-inhibitors/ARBs and beta-blockers is very prevalence in about four out of every five HFmEF patients while device therapy is very uncommon, use in less than $5 \%$ of $\mathrm{HFmEF}$ patients.

Individual sub analysis of three clinical registries provide insight into the effect of HF medication on mortality and hospitalization. In the CHART-2 registry, beta-blockers improved mortality while diuretics had a negative impact on prognosis in HFmEF and HFrEF patients but not HFpEF [29]. In the Swedish HF registry, ACE-inhibitors $(\mathrm{HR}=0.67) / \mathrm{ARBs}(\mathrm{HR}=0.59)$ improved mortality, beta-blockers improved mortality but only on the presence of CAD $(\mathrm{HR}=0.74, \mathrm{p}=$ 0.01 ) while diuretics had a negative effect on prognosis [16]. In TIMECHF beta-blockers improved mortality in HFmEF patients but to only those without AF (HR 0.48; 95\% CI: 0.24-0.97) [43]. The effect of HF medication on mortality and hospitalization were also reported in two large programs. Post hoc analysis of the Treatment of Preserved Cardiac Function Heart Failure with an Aldosterone Antagonist Trial (TOPCAT) registry in patients with LVEF $44 \%$ to $50 \%$ revealed MRAs improved composite HF-related-related death, aborted cardiac arrest and hospitalization $(\mathrm{HR}=0.72, \mathrm{p}=0.046)$ [44]. Post hoc analysis of the Candesartan in Heart Failure: Assessment of Reduction in Mortality and Morbidity (CHARM) registry that MRAs improved composite cardiovascular death or HF-hospitalization $(\mathrm{HR}=0.76, \mathrm{p}<0.02)$ [45].

Clinical trials have also indicated possible relationship between biomarkers and underlying etiology, and medication efficacy in HFmEF patients. In analysis of serum natriuretic peptides, Savarese et al. [35] associated the reduction of NT-proBNP levels in HFmEF patients with reduced risk of all-cause mortality or hospitalization. However, whether changes in the serum levels of NT-proBNP predict medication efficacy has not been demonstrated. The effect of the presence of CAD [16] and AF [43] on beta-blocker efficacy [16] suggest the importance of determining the underlying etiology to inform the choice of treatment since most underlying etiologies already have validated and approved treatment. Divergent outcomes on the effect of HF medication on HFmEF patients warrants clinical trials to confirm the benefits of HF therapies on HFmEF patients while taking into account underlying etiology and the levels of serum natriuretic peptides.

\section{Conclusion}

Heart failure with mid-rage ejection fraction (HFmEF) is the latest described phenotype of heart failure (HF). The previous ones are HF with reduced (HFrEF) and preserved ejection fraction (HFpEF). Since most clinical trials involve HFrEF and HFpEF phenotypes, limied data is available on the HFmEF phenotype. Clinical registries remain the best source of data to study the natural history, epidemiology, comorbidity burden, presentation, diagnosis and clinical management. Comorbidity burden of HFmEF is intermediate between HFrEF and HFpEF. It has a high prevalence of hypertension, atrial fibrillation and vascular disease comparable to HFpEF, and coronary artery disease and previous myocardial infarction comparable to HFrEF. Independent prognostic predictors are older age, male gender, higher systolic blood pressure, presence of diabetes mellitus, a history of myocardial infarction, and changes in the levels of natriuretic peptides. HFmEF has the highest rate of temporal LVEF changes, where transitioning to HFrEF predict worse prognosis while transitioning to HFpEF predict a favorable prognosis. In terms of etiology, HFmEF is an intermediate phenotype with ischemic etiology similar to $\mathrm{HFrEF}$ and vascular/ hypertensive etiology similar to HFpEF. Pathophysiologic mechanisms of HFmEF are unknown but levels of natriuretic peptides lying between that of HFrEF and HFpEF suggest a combination of myocardial stretch and inflammation. Typical clinical signs and symptoms such dyspnea, fatigue and peripheral edema are non-specific and cannot help distinguish the three HF phenotypes. Diagnosis is confirmed if there is presence of signs and/or symptoms, mildly depressed LVEF (40$49 \%$ ), elevated levels of natriuretic peptides, and evidence of structural heart disease (left ventricular hypertrophy or left atrial enlargement) or diastolic dysfunction. Clinical management adopts strategies validated for HFpEF. Conventional HF medication is the most common therapy while device therapy using implantable cardioverter-defibrillator and/or cardiac resynchronization therapy is very limited. Reduced natriuretic peptides may predict medication efficacy while the presence of comorbidities such as coronary artery disease and atrial fibrillation may affect medication efficacy. This, additional studies are warranted to determine HF medication efficacy in HFpEF patients while considering the effect of natriuretic peptides and underlying etiologies.

\section{References}

1. McMurray JJ, Adamopoulos S, Anker SD, Auricchio A, Bohm M, et al. (2012) ESC Guidelines for the diagnosis and treatment of acute and chronic heart failure 2012. Eur $J$ Heart Fail 14: 803-869. [Crossref]

2. Yancy CW, Jessup M, Bozkurt B, Butler J, Casey DE, et al. (2013) 2013 ACCF/AHA guideline for the management of heart failure: a report of the American College of Cardiology Foundation/American Heart Association Task Force on Practice Guidelines. $J$ Am Coll Cardiol 62: e147-e239. [Crossref]

3. Ponikowski P, Voors AA, Anker SD, Bueno H, Cleland JG, et al. (2016) 2016 ESC Guidelines for the diagnosis and treatment of acute and chronic heart failure: The Task Force for the diagnosis and treatment of acute and chronic heart failure of the European Society of Cardiology (ESC) Developed with the special contribution of the Heart Failure Association (HFA) of the ESC. Eur Heart J 37: 2129-2200. [Crossref]

4. Sidebotham D, Le Grice IJ (2007) Physiology and pathophysiology - clinica assessment of cardiac function: Stroke volume and ejection fraction. In Cardiothoracic Critical Care (p. 3-27). Butterworth-Heinemann Elsevier, Philadelphia, PA. [Crossref]

5. Borlaug BA, Paulus WJ (2010) Heart failure with preserved ejection fraction: pathophysiology, diagnosis, and treatment. Eur Heart J 32: 670-679. [Crossref]

6. Borlaug BA, Redfield MM (2011) Diastolic and systolic heart failure are distinct phenotypes within the heart failure spectrumresponse to borlaug and redfield. Circ 123: 2006-2014. [Crossref]

7. Linde C, Curtis AB, Fonarow GC, Lee K, Little W, et al. (2016) Cardiac resynchronization therapy in chronic heart failure with moderately reduced left ventricular ejection fraction: Lessons from the Multicenter InSync Randomized Clinical Evaluation MIRACLE EF study. Int J Cardiol 202:349-355. [Crossref]

8. Hsu JJ, Ziaeian B, Fonarow GC (2017) Heart failure with mid-range (borderline) ejection fraction: Clinical implications and future directions. JACC Heart Fail 5: 763 771. [Crossref] 
9. Solomon SD, Anavekar N, Skali H, McMurray JJ, Swedberg K, et al. (2005) Influence of ejection fraction on cardiovascular outcomes in a broad spectrum of heart failure patients. Circ 112: 3738-3744. [Crossref]

10. Lam CS, Solomon SD (2014) The middle child in heart failure: heart failure with midrange ejection fraction (40-50\%). Eur J Heart Fail 16: 1049-1055. [Crossref]

11. Gottdiener JS, McClelland RL, Marshall R, Shemanski L, Furberg CD, et al. (2002) Outcome of congestive heart failure in elderly persons: influence of left ventricular systolic function: the Cardiovascular Health Study. Ann Intern Med 137: 631-639. [Crossref]

12. He KL, Burkhoff D, Leng WX, Liang ZR, Fan L, et al. (2009) Comparison of ventricular structure and function in Chinese patients with heart failure and ejection fractions $>$ $55 \%$ versus $40 \%$ to $55 \%$ versus $<40 \%$. Am J Cardiol 103: $845-851$. [Crossref]

13. Cheng RK, Cox M, Neely ML, Heidenreich PA, Bhatt DL, et al. (2014) Outcomes in patients with heart failure with preserved, borderline, and reduced ejection fraction in the Medicare population. Am Heart J 168:721-730. [Crossref]

14. Kapoor JR, Kapoor R, Ju C, Heidenreich PA, Eapen ZJ, et al. (2916) Precipitating clinical factors, heart failure characterization, and outcomes in patients hospitalized with heart failure with reduced, borderline, and preserved ejection fraction. JACC Heart Fail 4: 464-472. [Crossref]

15. Chioncel O, Lainscak M, Seferovic PM, Anker SD, Crespo-Leiro MG, et al. (2017) Epidemiology and one-year outcomes in patients with chronic heart failure and preserved, mid-range and reduced ejection fraction: an analysis of the ESC Heart Failure Long-Term Registry. Eur J Heart Fail 19: 1574-1585. [Crossref]

16. Koh AS, Tay WT, Teng TH, Vedin O, Benson L, et al. (2017) A comprehensive population-based characterization of heart failure with mid-range ejection fraction. Eur $J$ Heart Fail 19: 1624-34. [Crossref]

17. Lofman I, Szummer K, Dahlstrom U, Jernberg T, Lund LH (2017). Associations with and prognostic impact of chronic kidney disease in heart failure with preserved, midrange, and reduced ejection fraction. Eur J Heart Fail 19: 1606-1614. [Crossref]

18. Rickenbacher P, Kaufmann BA, Maeder MT, Bernheim A, Goetschalckx K, et al. (2017) Heart failure with mid-range ejection fraction: a distinct clinical entity? Insights from the Trial of Intensified versus standard Medical therapy in Elderly patients with Congestive Heart Failure (TIME-CHF). Eur J Heart Fail 19: 1586-1596. [Crossref]

19. Bhambhani V, Kizer JR, Lima JA, van der Harst P, Bahrami H, et al. (2018) Predictors and outcomes of heart failure with mid-range ejection fraction. Eur J Heart Fail 20: 651-659. [Crossref]

20. Farre N, Lupon J, Roig E, Gonzalez-Costello J, Vila J, et al. (2017) Clinical characteristics, one-year change in ejection fraction and long-term outcomes in patients with heart failure with mid-range ejection fraction: a multicentre prospective observational study in Catalonia (Spain). BMJ 7: e018719. [Crossref]

21. Farmakis D, Simitsis P, Bistola V, Triposkiadis F, Ikonomidis I, et al. (2017) Acute hear failure with mid-range left ventricular ejection fraction: clinical profile, in-hospital management, and short-term outcome. Clinical Research in Cardiology 106: 359-368. [Crossref]

22. Guisado-Espartero ME, Salamanca-Bautista P, Aramburu-Bodas Ó, Conde-Martel A, Arias-Jiménez JL, et al. (2018) Heart failure with mid-range ejection fraction in patients admitted to internal medicine departments: Findings from the RICA Registry. Int. J. Cardiol 255: 124-128. [Crossref]

23. Pascual-Figal DA, Ferrero-Gregori A, Gomez-Otero I, Vazquez R, Delgado-Jimenez J, et al. (2017) Mid-range left ventricular ejection fraction: clinical profile and cause of death in ambulatory patients with chronic heart failure. Int. J. Cardiol 240: 265-270. [Crossref]

24. Gómez-Otero I, Ferrero-Gregori A, Román AV, Amigo JS, Pascual-Figal DA, et al. (2017) Mid-range ejection fraction does not permit risk stratification among patients hospitalized for heart failure. Revista Española de Cardiología (English Edition) 70: 338-346. [Crossref]

25. Lopatin Y (2018) Heart Failure with Mid-Range Ejection Fraction and How to Treat It. Cardiac failure review 4: 9-13. [Crossref]

26. Andronic AA, Mihaila S, Cinteza M (2016) Heart failure with mid-range ejection fraction: A new category of heart failure or still a gray zone. Maedica - a Journal of Clinical Medicine, 11: 320-324. [Crossref]

27. Nauta JF, Hummel YM, van Melle JP, van der Meer P, Lam CS, et al. (2017) What have we learned about heart failure with mid-range ejection fraction one year after its introduction? Eur J Heart Fail 19: 1569-1573. [Crossref]

28. Lam CS, Teng THK (2016) Understanding heart failure with mid-range ejection fraction. JACC Heart Fail 4: 473-476. [Crossref]
29. Tsuji K, Sakata Y, Nochioka K, Miura M, Yamauchi T, et al. (2017) Characterization of heart failure patients with mid-range left ventricular ejection fraction: a report from the Chart-2 Study. Eur J Heart Fail 19: 1258-1269. [Crossref]

30. Rastogi A, Novak E, Platts AE, Mann DL (2017) Epidemiology, pathophysiology and clinical outcomes for heart failure patients with a mid-range ejection fraction. Eur $J$ Heart Fail 19: 1597-1605. [Crossref]

31. Nadruz W, Junior EW, Santos M, Skali H, Groarke JD, et al. (2016) Heart Failure and Mid-Range Ejection Fraction: Implications of Recovered Ejection Fraction for Exercise Tolerance and Outcomes. Circ Heart Fail 9: e002826. [Crossref]

32. Clarke CL, Grunwald GK, Allen LA, Barón AE, Peterson PN, et al. (2013) Natura history of left ventricular ejection fraction in patients with heart failure. Circ Cardiovasc Qual Outcomes 6: 680-686. [Crossref]

33. Tromp J, Khan MA, Mentz RJ, O'Connor CM, Metra M, et al. (2017) Biomarker profiles of acute heart failure patients with a mid-range ejection fraction. JACC Heart Fail 5: 507-517. [Crossref]

34. Gohar A, Chong JP, Liew OW, den Ruijter H, de Kleijn DP, et al. (2017) The prognostic value of highly sensitive cardiac troponin assays for adverse events in men and women with stable heart failure and a preserved vs. reduced ejection fraction. Eur J Heart Fail 19: 1638-1647. [Crossref]

35. Savarese G, Hage C, Orsini N, Dahlström U, Perrone-Filardi P, et al. (2016) Reductions in N-terminal pro-brain natriuretic peptide levels are associated with lower mortality and heart failure hospitalization rates in patients with heart failure with mid-range and preserved ejection fraction. Circ Heart Fail 9: e003105. [Crossref]

36. He KL, Burkhoff D, Leng WX, Liang ZR, Fan L, et al. (2009) Comparison of ventricular structure and function in Chinese patients with heart failure and ejection fractions $>$ $55 \%$ versus $40 \%$ to $55 \%$ versus $<40 \%$. Am J Cardiol 103: 845-851. [Crossref]

37. McMurray JJ, Adamopoulos S, Anker SD, Auricchio A, Böhm M, et al. (2012) ESC Guidelines for the diagnosis and treatment of acute and chronic heart failure 2012: The Task Force for the Diagnosis and Treatment of Acute and Chronic Heart Failure 2012 of the European Society of Cardiology. Developed in collaboration with the Heart Failure Association (HFA) of the ESC. Eur J Heart Fail 14: 803-869. [Crossref]

38. Pit B, Zannad F, Remme WJ, Cody R, Castaigne A, et al. (1999) The effect of spironolactone on morbidity and mortality in patients with severe heart failure. $N$ Engl JMed 341: 709-717. [Crossref]

39. Kadish A (2004) Defibrillators in non-ischemic cardiomyopathy treatment evaluation (DEFINITE) investigators: Prophylactic defibrillator implantation in patients with nonischemic dilated cardiomyopathy. N Engl J Med 350: 2151-2158. [Crossref]

40. Goldenberg I, Moss AJ, Hall WJ, McNitt S, Zareba W, et al. (2006) Causes and consequences of heart failure after prophylactic implantation of a defibrillator in the multicenter automatic defibrillator implantation trial II. Circulation 113: 2810-2817. [Crossref]

41. Parissis J, Farmakis D, Triposkiadis F (2016) Heart failure registries: how far can we go? Eur J Heart Fail 18: 626-628. [Crossref]

42. Shah KS, Xu H, Matsouaka RA, Bhatt DL, Heidenreich PA, Hernandez AF, et al. (2017) Heart failure with preserved, borderline, and reduced ejection fraction: 5-year outcomes. J Am Coll Cardiol 70: 2476-2486. [Crossref]

43. Cleland JG, Bunting KV, Flather MD, Altman DG, Holmes J, et al. (2017) Betablockers for heart failure with reduced, mid-range, and preserved ejection fraction: an individual patient-level analysis of double-blind randomized trials. Eur Heart $J 39$ : 26-35. [Crossref]

44. Solomon SD, Claggett B, Lewis EF, Desai A, Anand I, Sweitzer NK, O'meara E, Shah SJ, McKinlay S, Fleg JL, Sopko G. Influence of ejection fraction on outcomes and efficacy of spironolactone in patients with heart failure with preserved ejection fraction. Eur Heart J37: 455-462. [Crossref]

45. Lund LH, Claggett B, Liu J, Lam CS, Jhund PS, et al. (2018) Heart failure with midrange ejection fraction in CHARM: characteristics, outcomes and effect of candesartan across the entire ejection fraction spectrum. Eur J Heart Fail 20: 1230-1239 [Crossref]

Copyright: (C)2018 Albakri A. This is an open-access article distributed under the terms of the Creative Commons Attribution License, which permits unrestricted use, distribution, and reproduction in any medium, provided the original author and source are credited. 